\title{
The Research on Integrated Communication Networks and Satellite Nav- igation Positioning Technology
}

\author{
Jie Xia ${ }^{1, \text { a }}$, Zhongliang Deng ${ }^{1, a}$, Li Gao ${ }^{1, \text { a }}$ \\ ${ }^{1}$ Beijing University of Post and Telecommunication, Beijing 100876, China \\ axiajie_offer@163.com
}

\begin{abstract}
Keywords: Communication Networks; Satellite Navigation; Ranging Error; Integrated Positioning
\end{abstract} Technology; Precise Ranging.

\begin{abstract}
This paper mainly compares and analyses ranging technology of communication signals and navigation signals respectively, and introduces the integration of terrestrial mobile communication base station navigation and positioning technology. Also it focuses on how to achieve precisely ranging based on the integration of communication signals and navigation signals, which is in order to improve the positioning accuracy. With theoretical analysis and software simulation, we improve the integrated signals multipath suppression ability and achieve the precise ranging technology.
\end{abstract}

\section{Introduction}

The past 10 years, as a national high-tech strategic new industry, location based severs (LBS) begins to integrate into all fields of life. According to statistics, $80 \%$ of information people use is related to the "position". In the field of national security, disaster relief, counter-terrorism operations, elderly care, smart city construction, location information needs much more urgently. Therefore, high-precision positioning and navigation technology has become a hot research field in recent years. It is not only an important foundation to support location-based services industry, but also the most important means to obtain the location information. Range finding technology is the most direct way to obtain location information of an object, but also the basis of the mobile terminal positioning solver. Rough range finding errors will result in uncertainty of location compensation, which is directly related to the level of positioning accuracy. Therefore, the study of the precise range finding technology is of great significance for enhancing the positioning accuracy. In this paper, based on this background, we study the ranging technology with integration of communication signals and navigation signals, which results in improving the overall signal ranging accuracy.

\section{The Research Status at Home and Abroad}

\subsection{Pseudo-code Ranging Method}

Pseudo-code ranging method is a common radio ranging method. Pseudo-random code which is commonly used include sequence of M, Walsh Sequences, Gold Sequences and RS Sequences. The basic principle of this method is that the terminal use local pseudo-random code to do correlation calculation after the capture of pseudo-random code signals, so as to achieve the purpose of tracking and ranging signals. But this method requires the period of the code sequence is greater than the signal cycle round delay, so as to ensure ambiguity resolution. Meanwhile, in order to accurately capture and tracking, it requires sufficiently sharp autocorrelation peak and small symbol width.

\subsection{Carrier Phase Ranging Method}

Carrier phase ranging method is a superior performance ranging method, the main principle of which is that by observing and measuring the phase difference of the satellite carrier signal and local oscillator reference signal, then calculating signal transmission time, thus achieving range finding. Assuming the transmitted signal can be expressed as:

$$
s(t)=a e^{j 2 \pi f_{1} t}
$$

a represents the amplitude, $f_{1}$ represents signal frequency. After spatial propagation time of $\tau$, received signal can be expressed as: 


$$
r(t)=\sqrt{\xi} e^{j 2 \pi f_{1}(t+\tau)}+n(t)
$$

$\xi$ represents signal power attenuation, $n(t)$ represents additive white Gaussian noise. The signal transmission time $\Delta t$ can be expressed as:

$$
\Delta t=\left(\phi_{r}-\phi_{s}\right) / 2 \pi f_{1}
$$

$\phi_{r}$ and $\phi_{S}$ respectively represents transmitted and received signal phase of the signal.

The carrier phase ranging produces phase ambiguity problem, making a significant deviation ranging. However, there is no good solution for this problem.

\subsection{Time Difference of Arrival (TDOA) Ranging Method}

TDOA ranging method is more popular in recent years, which mainly uses value of the signal arrival time (Time of Arrival, TOA) to calculate pseudo-range, and then implementing location solver with distance intersection method. This method requires only three mobile base stations or satellites to locate, which applies more widely.

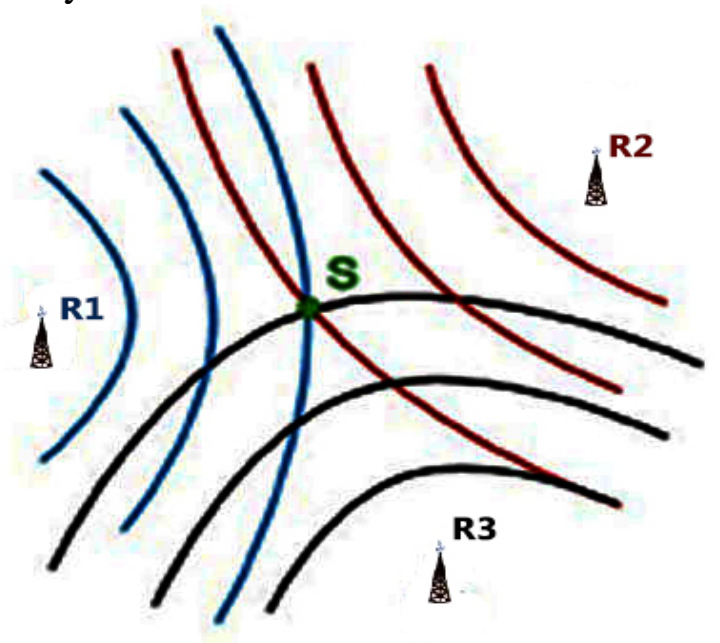

Fig.1 TDOA positioning diagram

\section{Communication signals ranging of LTE}

Orthogonal frequency-division multiplexing (OFDM) is a signal system using in LTE, which has a high spectral efficiency and excellent resistance to multipath fading and anti-fading. It is often used in terrestrial mobile communications applications. This section studies the ranging method with OFDM signals and ranging performance analysis in multipath environments.

\subsection{Ranging Principle of OFDM}

In current OFDM system, at the transmitting end, the transport block after channel coding, modulation, precoding and other processes, by Inverse Fast Fourier Transform (IFFT) transmitted information is mapped to different subcarriers. At the receiving end through the reverse process, information will be demodulated. The figure below is the basic model diagram OFDM system.
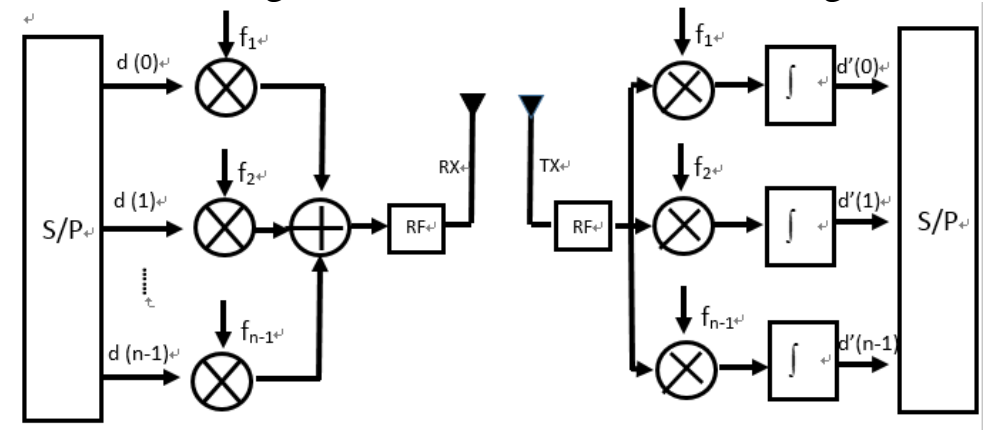

Fig.2 OFDM system model diagram

Transmitted signal in OFDM systems can be expressed as: 


$$
S(t)=\frac{1}{N} \sum_{i=0}^{i=N-1} C_{i} \exp \left(j 2 \pi \frac{i}{T} t\right), \quad 0 \leq t \leq T
$$

$C_{i}$ is the data information in OFDM symbol, $N$ is the number of subcarriers, $T$ is the duration of the symbol. In the OFDM signal regime, there is synchronizing signal provided in a radio frame. The synchronization signal transmit known code sequence, on which the receiver can be based for system synchronization and ranging.

OFDM ranging sufficiently uses of the OFDM signal's time-frequency characteristics. In the time domain, correlation operation of the received signal obtains a rough time delay estimation. In the frequency domain, when the time of the max correlation value arrives, using the FFT transform, which obtains the delay caused by phase shift, then we can get an accurate estimation.

\subsection{OFDM Ranging Performance Simulation}

Based on the above theoretical analysis, in this section, computer simulation is implemented in multipath environments. In the simulation, parameters are set as followings: OFDM bandwidth of $20 \mathrm{MHz}$, the number of subcarriers is 4096 , the sampling frequency is $20 \mathrm{MHz}$. The simulation results are shown as follows:

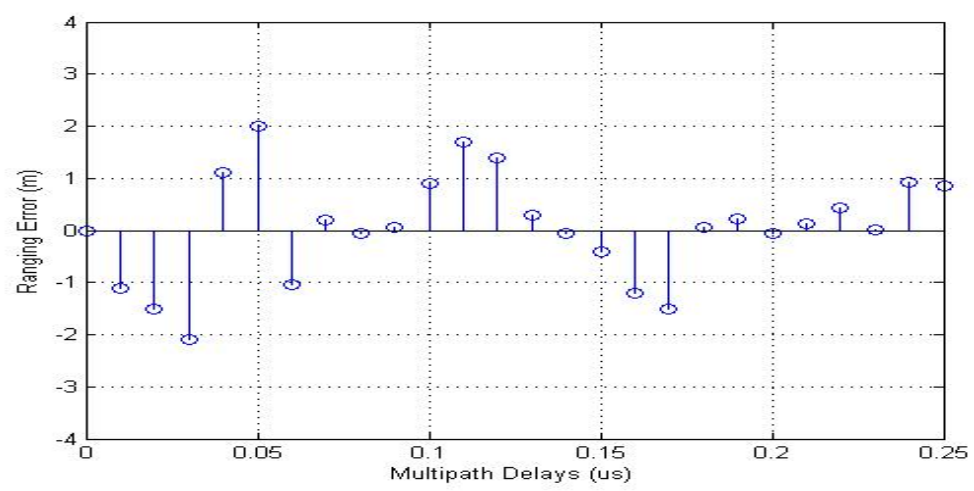

Fig.3 OFDM ranging multipath error

From the figure, we can see that OFDM ranging error shows cyclical fluctuations with multipath delays.

\section{Navigation Signals Ranging}

Duing to better noise immunity and autocorrelation, code division multiple access capabilities, in satellite positioning systems, CDMA signals are used to implement accurate pseudo ranging in condition of weak signal. This section studies the principles of CDMA signals ranging in multipath environments and its performance analysis.

\subsection{Ranging Principle of CDMA}

Ranging with CDMA signals, firstly, rough synchronization of code phase and carrier frequency is implemented, which can acquire pseudo-code. Thereby obtaining a rough delay and carrier frequency signals. Thereafter, code loop and carrier ring clone received signal, whereby the received signal is accurately synchronized to achieve accurate estimation of the propagation delay and code phase. Wherein the code loop phase detector output as the basis for the adjustment ring code, which directly affects the accuracy range accuracy CDMA signal.

In the CDMA ranging signal, the code loop is generally duplicated three different phases of the local code, respectively: Early, Prompt, Late. And then implementing correlation with the received digital signal, which obtains E, P, L. Taking into account the plurality of code loop discriminator, only the early and late two associated support on the road as an input signal, the phase detector based on the results of $\mathrm{E}$ and $\mathrm{L}$ to calculate the fractional part of the code phase delay, commonly calculated formula is as follows:

$$
\delta=(1-d) \frac{E-L}{E+L}
$$

$d$ is the correlator spacing. 


\subsection{CDMA Ranging Performance Simulation}

Based on the above theoretical analysis, this section implement ranging simulation of the CDMA signal. The parameters are set as followings: CDMA code rate is $10 \mathrm{MHz}$, the chip width is $100 \mathrm{~ns}$, the correlator spacing of 0.2 and 0.5 , respectively, using two types of parameters. In the same or opposite path multipath signal level and the first positive and negative characteristics of the situation was simulated, the results are as follows:

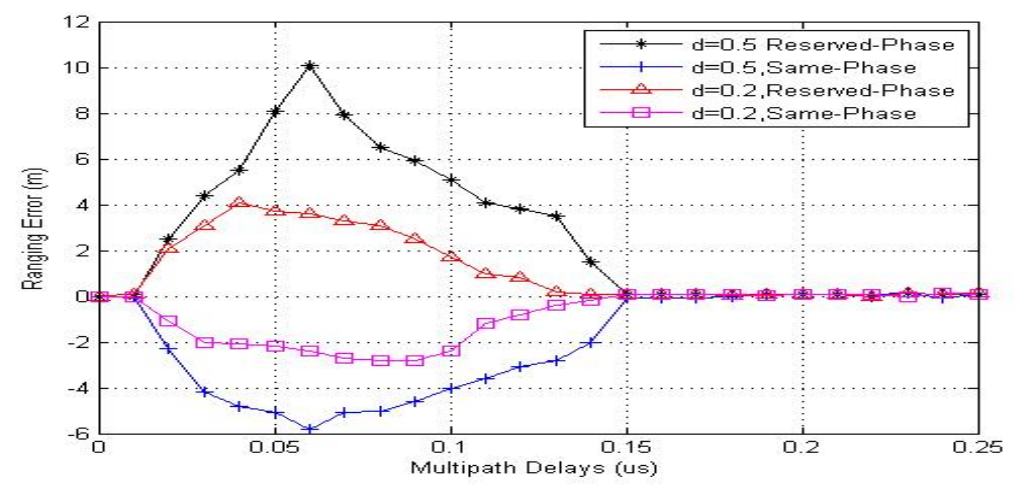

Fig.4 CDMA ranging multipath error

As can be seen from the figure, when the multi-path delay is small, effects of multipath CDMA ranging by more severe; when multi-path delay is large, CDMA ranging is no longer affected by multipath, but OFDM Ranging multipath interference still exists.

\section{Integrated Communication Signals and Navigation Signals Ranging}

Through the above analysis, OFDM signal multipath delay ranging fluctuates periodically, there is no obviously good or bad; CDMA ranging in large delay is better performance, worse when small. This section mainly studies integration of OFDM signals and CDMA signals ranging and performance simulation.

\subsection{Ranging Principle of Integrated Signal}

Integrated signal ranging process is as follows:

(1) Acquiring coarse code phase and carrier frequency with OFDM signal, providing the initial correlation calculation phase and carrier frequency for the CDMA signal;

(2) Calculating and tracking CDMA signal for a long period of correlation, access to more sophisticated code phase and carrier frequency information;

(3) With frequency synchronization information obtained in accordance with the precise time, calculating accurate delay with the OFDM ranging signal;

(4) Accurate delay measurements obtained in OFDM ranging feedback to CDMA tracking loop, which can enhance the range accuracy with CDMA signal.

From sections III and IV, it can be seen the OFDM signal has small ranging error in a small multi-path delay, but well-CDMA signal multipath delay ranging in performance is large multi-path delay, that is CDMA has a strong resolution outside the multipath CDMA chip. Using the advantages of both, to achieve a mutually reinforcing OFDM and CDMA signal, complementary advantages, enhance the integration of the signal range accuracy.

\subsection{Integrated Signal Ranging Performance Simulation}

According to the theoretical analysis, this section focuses on the integrated signal ranging simulation, the simulation parameters are set as follows: CDMA and OFDM signal system bandwidth are $20 \mathrm{MHz}$, CDMA rate of $10 \mathrm{MHz}$, the number of OFDM subcarriers 4096, the sampling frequency is $20 \mathrm{MHz}$, multipath interval is $0.01 \mathrm{us}$, the correlator spacing is set to 0.2 , and the simulation results are shown below: 


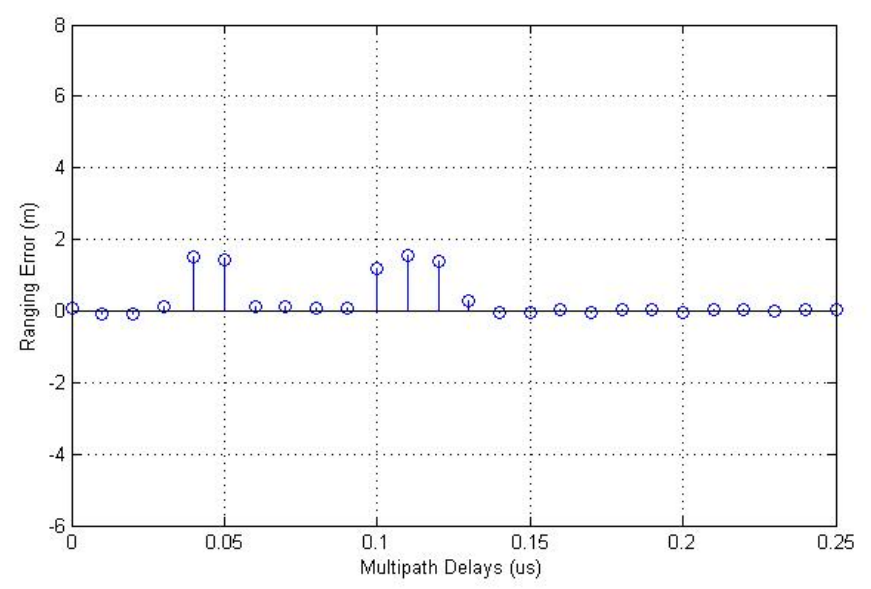

Fig.6 Integrated signal ranging multipath error

From the figure, the integrated signal has greatly improved compared with the OFDM and CDMA in ranging error, reaching the communication signals ranging and navigation signals ranging mutually reinforcing, complementary effects and improve the positioning accuracy.

\section{Conclusion}

This paper studies ranging problems in the navigation process, a method of integration of communication signals and navigation signals ranging is proposed. Theoretical analysis and simulation results prove that this method has greater enhance in signal multipath environments ranging accuracy, which is good for the overall positioning accuracy. I believe that, with the rapid development of mobile Internet, high-precision positioning and navigation technology will be the basis for the location of the service industry, and the signal ranging technology is a key factor restricting the development of high-precision positioning and navigation technology. Therefore, this paper studies the technical basis for the location of the service industry has an important significance.

\section{Acknowledgements}

This work was supported by the National High Technology Research and Development Program (863 Program) of China under Grant No.2015AA124101; the National High Technology Research and Development Program (863 Program) of China under Grant No.2014AA123103; the National Natural Science Foundation of China under Grant No.61401040, and the National Natural Science Foundation of China under Grant No.61372110.

\section{References}

[1]. Chen shijin. High Precision Ranging radio engineering. 2003, vol.33. p. 22-23.

[2]. Guo qing, Zeng hongBo. Analyzed the satellite monitoring system in a pseudo-random code ranging method of communication technologies. 2003, vol.10.p.505-509.

[3]. Xie Gang. GPS receiver design principles and Beijing: Electronic Industry Press, 2009.7

[4]. R. Van Nee and R. Prasad OFDM for Multimedia Communications Boston:. Artech House, 2000.

[5]. Zhang huiNing, Ma ying, REN Guang-liang, etc. Ranging study of orthogonal frequency division multiplexing method with detection system. Detection \& Control. 2005, vol.27. p. 16-18.

[6]. Yu yanpei. Study on the key Technology of TC-OFDM for High Precision Indoor Positioning Based on Mobile Base Station. 2013.4 